\title{
Modules of differential operators of order 2 on Coxeter arrangements
}

\author{
Norihiro Nakashima
}

\begin{abstract}
The collection of reflection hyperplanes of a finite reflection group is called a Coxeter arrangement. A Coxeter arrangement is known to be free. K. Saito has constructed a basis consisting of invariant elements for the module of derivations on a Coxeter arrangement. We study the module of $\mathscr{A}$-differential operators as a generalization of the study of the module of $\mathscr{A}$-derivations. In this article, we prove that the modules of differential operators of order 2 on Coxeter arrangements of types A, B and D are free, by exhibiting their bases. We also prove that the modules cannot have bases consisting of only invariant elements. Two keys for the proof of freeness are "Cauchy-Sylvester's theorem on compound determinants" and "Saito-Holm's criterion."
\end{abstract}

Key Words: Coxeter arrangement, Cauchy-Sylvester's compound determinants, Schur functions.

2010 Mathematics Subject Classification: Primary 32S22, Secondary $15 \mathrm{~A} 15$.

\section{Introduction}

Let $V=\mathbb{R}^{\ell}$ be a Euclidean space of dimension $\ell$ over $\mathbb{R}$. Let $\left\{x_{1}, \ldots, x_{\ell}\right\}$ be a basis for the dual space $V^{*}$, and let $S:=\operatorname{Sym}\left(V^{*}\right) \simeq \mathbb{R}\left[x_{1}, \ldots, x_{\ell}\right]$ be the polynomial ring. Put $\partial_{i}:=\frac{\partial}{\partial x_{i}}$ for $i=1, \ldots, \ell$. Let $D^{(m)}(S):=\bigoplus_{|\boldsymbol{\alpha}|=m} S \partial^{\boldsymbol{\alpha}}$ be the module of differential operators (of order $m$ ) of $S$, where $|\boldsymbol{\alpha}|:=\alpha_{1}+\cdots+\alpha_{\ell}$ and $\partial^{\alpha}:=\partial_{1}^{\alpha_{1}} \cdots \partial_{\ell}^{\alpha_{\ell}}$ for a multi-index $\boldsymbol{\alpha}=\left(\alpha_{1}, \ldots, \alpha_{\ell}\right) \in \mathbb{N}^{\ell}$. A nonzero element $\theta=\sum_{|\boldsymbol{\alpha}|=m} f_{\boldsymbol{\alpha}} \partial^{\boldsymbol{\alpha}} \in D^{(m)}(S)$ is homogeneous of degree $i$ if $f_{\boldsymbol{\alpha}}$ is zero or homogeneous of degree $i$ for each $\boldsymbol{\alpha}$. When $\theta \in D^{(m)}(S)$ is homogeneous of degree $i$, we write $\operatorname{deg}(\theta)=i$. For a multi-index $\boldsymbol{\alpha}$, 
we put

$$
\begin{aligned}
x_{\boldsymbol{\alpha}} & :=\left(x_{1}, \ldots, x_{1}, x_{2}, \ldots, x_{2}, \ldots, x_{\ell}, \ldots, x_{\ell}\right), \\
x_{\boldsymbol{\alpha}}^{2} & :=\left(x_{1}^{2}, \ldots, x_{1}^{2}, x_{2}^{2}, \ldots, x_{2}^{2}, \ldots, x_{\ell}^{2}, \ldots, x_{\ell}^{2}\right)
\end{aligned}
$$

where the number of $x_{i}$ (or $\left.x_{i}^{2}\right)$ is $\alpha_{i}$.

Let $\mathscr{A}$ be a central (hyperplane) arrangement (i.e., every hyperplane contains the origin) in $V$. Fix a linear form $p_{H} \in V^{*}$ such that $\operatorname{ker}\left(p_{H}\right)=H$ for each hyperplane $H \in \mathscr{A}$, and put $Q(\mathscr{A}):=\prod_{H \in \mathscr{A}} p_{H}$. We call $Q(\mathscr{A})$ a defining polynomial of $\mathscr{A}$. We define the module $D^{(m)}(\mathscr{A})$ of $\mathscr{A}$-differential operators of order $m$ by

$$
D^{(m)}(\mathscr{A}):=\left\{\theta \in D^{(m)}(S) \mid \theta(Q(\mathscr{A}) S) \subseteq Q(\mathscr{A}) S\right\}
$$

In the case $m=1, D^{(1)}(\mathscr{A})$ is the module of $\mathscr{A}$-derivations. We say $\mathscr{A}$ to be free if $D^{(1)}(\mathscr{A})$ is a free $S$-module. An excellent reference on arrangements is the book by Orlik and Terao [10].

For a commutative $\mathbb{R}$-algebra $R$, let $\mathscr{D}(R)$ denote the ring of differential operators. Then $\mathscr{D}(S)$ is the Weyl algebra. For an ideal $J$ of $S$, let $\mathscr{D}(J)$ denote the subring of $\mathscr{D}(S)$ consisting of the operators preserving the ideal $J$. There is a ring isomorphism:

$$
\mathscr{D}(S / J) \simeq \mathscr{D}(J) / J \mathscr{D}(S)
$$

(see [7, Theorem 15.5.13]). Holm [3] showed that $\mathscr{D}(Q(\mathscr{A}) S)$ decomposes into the direct sum of $D^{(m)}(\mathscr{A})$. Thus we have an $S$-module isomorphism

$$
\mathscr{D}(S / Q(\mathscr{A}) S) \simeq \frac{\bigoplus_{m \geq 0} D^{(m)}(\mathscr{A})}{Q(\mathscr{A}) \mathscr{D}(S)} .
$$

There has been a lot of research on finiteness properties of rings of differential operators. Systems of generators for $\mathscr{D}(R)$ are usefull to study finiteness properties. For example, it is known that $\mathscr{D}(S / Q(\mathscr{A}) S)$ is a Noetherian ring when $\mathscr{A}$ is a 2dimensional central arrangement, and an expression by a basis played a key role in the proof of [8]. One of the aim to study freeness for the module $D^{(m)}(\mathscr{A})$ of $\mathscr{A}$ differential operators is to give an $S$-basis (or $S$-generators) of the ring of differential operators $\mathscr{D}(S / Q(\mathscr{A}) S)$. As the first step, we put the study of the module $D^{(2)}(\mathscr{A})$ into practice when $\mathscr{A}$ is a Coxeter arrangement.

Let $W$ be a finite reflection group generated by reflections acting on $V$. Naturally $W$ acts on $S$, and $W$ acts on the tensor products $D^{(m)}(S) \simeq S \otimes_{\mathbb{R}} \sum_{|\boldsymbol{\alpha}|=m} \mathbb{R} \partial^{\boldsymbol{\alpha}}$. The collection of reflection hyperplanes of $W$ is called a Coxeter arrangement (or 
a reflection arrangement). Coxeter arrangements $\mathcal{A}_{\ell-1}, \mathcal{B}_{\ell}$ and $\mathcal{D}_{\ell}$ are respectively defined by

$$
\begin{aligned}
& \mathcal{A}_{\ell-1}:=\left\{H_{i j}=\left\{x_{i}-x_{j}=0\right\} \mid 1 \leq i<j \leq \ell\right\}, \\
& \mathcal{B}_{\ell}:=\left\{H_{i}=\left\{x_{i}=0\right\} \mid i=1, \ldots, \ell\right\} \cup\left\{H_{i j}^{ \pm 1}=\left\{x_{i} \pm x_{j}=0\right\} \mid 1 \leq i<j \leq \ell\right\}, \\
& \mathcal{D}_{\ell}:=\left\{H_{i j}^{ \pm 1}=\left\{x_{i} \pm x_{j}=0\right\} \mid 1 \leq i<j \leq \ell\right\} .
\end{aligned}
$$

From now on, we assume that $\mathscr{A}$ is a Coxeter arrangement. The module of $\mathscr{A}$ derivations is related to the invariant theory of the reflection group corresponding to $\mathscr{A}$. K. Saito has proved that a Coxeter arrangement $\mathscr{A}$ is free, and the module of $\mathscr{A}$-derivations is isomorphic to $S \otimes_{S^{W}} D^{(1)}(S)^{W}$ as an $S$-module, where $S^{W}$ and $D^{(1)}(S)^{W}$ are the set of invariant elements of $S$ and $D^{(1)}(S)$, respectively (see, for example, Theorem 6.60 in [10]). In particular, there exists a basis for the module of $\mathscr{A}$-derivations consisting of invariant elements. We can find an explicit basis for $D^{(1)}(\mathscr{A})$ in [6] when $\mathscr{A}$ are Coxeter arrangements $\mathcal{A}_{\ell-1}, \mathcal{B}_{\ell}$ and $\mathcal{D}_{\ell}$. However, $D^{(2)}(\mathscr{A})$ cannot have bases consisting of only invariant elements when $\mathscr{A}$ are Coxeter arrangements $\mathcal{A}_{\ell-1}, \mathcal{B}_{\ell}$ and $\mathcal{D}_{\ell}$. We prove the assertion above in Section 6 .

In this paper we prove that the module of differential operators of order 2 on Coxeter arrangements $\mathcal{A}_{\ell-1}, \mathcal{B}_{\ell}$ and $\mathcal{D}_{\ell}$ are free by constructing bases in Section 4 and 5. For this purpose, we introduce Cauchy-Sylvester's theorem on compound determinants and Saito-Holm's criterion. In Section 3, we give some applications of the Cauchy-Sylvester's theorem on compound determinants.

The results of this work (without proofs) have been submitted as an extended abstract to FPSAC 2012 [9].

\section{Saito-Holm's criterion}

In this section, we explain Saito-Holm's criterion. Put $s_{m}:=\left(\begin{array}{c}\ell+m-1 \\ m\end{array}\right)$ and $t_{m}:=$ $\left(\begin{array}{c}\ell+m-2 \\ m-1\end{array}\right)$, and set

$$
\left\{\boldsymbol{\alpha}^{(1)}, \ldots, \boldsymbol{\alpha}^{\left(s_{m}\right)}\right\}=\left\{\boldsymbol{\alpha} \in \mathbb{N}^{\ell}|| \boldsymbol{\alpha} \mid=m\right\},
$$

where $|\boldsymbol{\alpha}|=\alpha_{1}+\cdots+\alpha_{\ell}$ for a multi-index $\boldsymbol{\alpha} \in \mathbb{N}^{\ell}$. For operators $\theta_{1}, \ldots, \theta_{s_{m}} \in$ $D^{(m)}(\mathcal{A})$, define the coefficient matrix $M_{m}\left(\theta_{1}, \ldots, \theta_{s_{m}}\right)$ of the operators $\theta_{1}, \ldots, \theta_{s_{m}}$ as follows:

$$
M_{m}\left(\theta_{1}, \ldots, \theta_{s_{m}}\right):=\left[\theta_{i}\left(\frac{x^{\boldsymbol{\alpha}^{(j)}}}{\boldsymbol{\alpha}^{(j) !}}\right)\right]_{1 \leq i, j \leq s_{m}},
$$


where $\boldsymbol{\alpha} !=\alpha_{1} ! \cdots \alpha_{\ell} !$. Thus the $(i, j)$-entry of the coefficient matrix is the polynomial coefficient of $\partial^{\boldsymbol{\alpha}^{(j)}}$ in $\theta_{i}$.

The following criterion was originally given by Saito [11] in the case $m=1$, and was generalized by Holm [2] into the case $m$ general.

Proposition 2.1 (Saito-Holm's criterion). Let $\theta_{1}, \ldots, \theta_{s_{m}} \in D^{(m)}(\mathscr{A})$ be homogeneous operators. Then the following two conditions are equivalent:

(1) $\operatorname{det} M_{m}\left(\theta_{1}, \ldots, \theta_{s_{m}}\right)=c Q^{t_{m}}$ for some $c \in \mathbb{R}^{\times}$.

(2) $\theta_{1}, \ldots, \theta_{s_{m}}$ form a basis for $D^{(m)}(\mathscr{A})$ over $S$.

When $D^{(m)}(\mathscr{A})$ is a free $S$-module, we define the exponents of $D^{(m)}(\mathscr{A})$ to be the multi-set of degrees of a homogeneous basis $\left\{\theta_{1}, \ldots, \theta_{s_{m}}\right\}$ for $D^{(m)}(\mathscr{A})$, which is denoted by $\exp D^{(m)}(\mathscr{A})$ :

$$
\exp D^{(m)}(\mathscr{A})=\left\{\operatorname{deg}\left(\theta_{1}\right), \ldots, \operatorname{deg}\left(\theta_{s_{m}}\right)\right\} .
$$

\section{Cauchy-Sylvester's theorem on compound de- terminants}

Throughout this paper, we assume $\ell \geq m$. In this section, we will follow the notation of the paper by Ito and Okada [5] as far as possible. We denote by $\succ$ the lexicographic order on $\mathbb{Z}^{m}$. That is, for $\mu=\left(\mu_{1}, \ldots, \mu_{m}\right)$ and $\nu=\left(\nu_{1}, \ldots, \nu_{m}\right) \in \mathbb{Z}^{m}$, we write $\mu \succ \nu$ if there exist an index $k$ such that

$$
\mu_{1}=\nu_{1}, \ldots, \mu_{k-1}=\nu_{k-1} \text {, and } \mu_{k}>\nu_{k} .
$$

Set

$$
Z:=\left\{\mu=\left(\mu_{1}, \ldots, \mu_{m}\right) \in \mathbb{Z}^{m} \mid 1 \leq \mu_{1}<\mu_{2}<\cdots<\mu_{m} \leq \ell\right\} .
$$

Then $Z$ is a totally ordered subset of $\mathbb{Z}^{m}$. Put $x_{\mu}:=\left(x_{\mu_{1}}, \ldots, x_{\mu_{m}}\right) \in S^{m}$.

Let $A=\left(a_{i, j}\right)_{1 \leq i, j \leq \ell}$ be a square matrix of order $\ell$. For $\mu, \nu \in Z$ put

$$
A_{\mu, \nu}:=\left(a_{\mu_{i}, \nu_{j}}\right)_{1 \leq i, j \leq m} .
$$

We define the $m$-th compound matrix $A^{(m)}$ by

$$
A^{(m)}:=\left(\operatorname{det} A_{\mu, \nu}\right)_{\mu, \nu \in Z},
$$


where the rows and columns are arranged in the increasing order on $Z$.

The following was obtained by Cauchy and Sylvester (see, for example, [5, Proposition 3.1]).

Proposition 3.1 (Cauchy-Sylvester). Let $A=\left(a_{i, j}\right)_{1 \leq i, j \leq \ell}$ be a square matrix. Then the determinant of the $m$-th compound matrix $A^{(m)}$ is given by

$$
\operatorname{det} A^{(m)}=(\operatorname{det} A)^{\left(\begin{array}{c}
\ell-1 \\
m-1
\end{array}\right)} .
$$

Put

$$
\Lambda:=\left\{\lambda=\left(\lambda_{1}, \ldots, \lambda_{m}\right) \in \mathbb{Z}^{m} \mid \ell-m \geq \lambda_{1} \geq \lambda_{2} \geq \cdots \geq \lambda_{m} \geq 0\right\} .
$$

We regard $\Lambda$ as a totally ordered subset of $\mathbb{Z}^{m}$ by the order $\succ$. Then the map

$$
Z \ni\left(\mu_{1}, \ldots, \mu_{m}\right) \longmapsto\left(\ell-m+1-\mu_{1}, \ell-m+2-\mu_{2}, \ldots, \ell-\mu_{m}\right) \in \Lambda
$$

is a bijection between $\Lambda$ and $Z$, and this bijection reverses the ordering on $\Lambda$ and $Z$.

For $\lambda \in \Lambda$, we define the following symmetric polynomials and a Laurent polynomial:

$$
\begin{aligned}
s_{\lambda}^{\mathcal{A}} & :=\frac{\operatorname{det}\left(t_{i}^{\lambda_{j}+m-j}\right)_{1 \leq i, j \leq m}}{\operatorname{det}\left(t_{i}^{m-j}\right)_{1 \leq i, j \leq m}} \in S\left[t_{1}, \ldots, t_{m}\right], \\
s_{\lambda}^{\mathcal{B}} & :=\frac{\operatorname{det}\left(t_{i}^{2\left(\lambda_{j}+m-j\right)+1}\right)_{1 \leq i, j \leq m}}{\operatorname{det}\left(t_{i}^{2(m-j)}\right)_{1 \leq i, j \leq m}} \in S\left[t_{1}, \ldots, t_{m}\right], \\
s_{\lambda}^{\mathcal{D}} & :=\frac{\operatorname{det}\left(t_{i}^{2\left(\lambda_{j}+m-j\right)-1}\right)_{1 \leq i, j \leq m}}{\operatorname{det}\left(t_{i}^{2(m-j)}\right)_{1 \leq i, j \leq m}} \in S\left[t_{1}^{ \pm 1}, \ldots, t_{m}^{ \pm 1}\right] .
\end{aligned}
$$

The polynomial $s_{\lambda}^{\mathcal{A}}$ is the Schur polynomial corresponding to the partition $\lambda$. The Laurent polynomials $s_{\lambda}^{\mathcal{B}}$ and $s_{\lambda}^{\mathcal{D}}$ may be expressed by $s_{\lambda}^{\mathcal{A}}$ as follows:

$$
\begin{aligned}
& s_{\lambda}^{\mathcal{B}}=t_{1} \cdots t_{m} \cdot \frac{\operatorname{det}\left(\left(t_{i}^{2}\right)^{\lambda_{j}+m-j}\right)_{1 \leq i, j \leq m}}{\operatorname{det}\left(\left(t_{i}^{2}\right)^{m-j}\right)_{1 \leq i, j \leq m}}=t_{1} \cdots t_{m} s_{\lambda}^{\mathcal{A}}\left(t_{1}^{2}, \ldots, t_{m}^{2}\right) \\
& s_{\lambda}^{\mathcal{D}}=\frac{1}{t_{1} \cdots t_{m}} \cdot \frac{\operatorname{det}\left(\left(t_{i}^{2}\right)^{\lambda_{j}+m-j}\right)_{1 \leq i, j \leq m}}{\operatorname{det}\left(\left(t_{i}^{2}\right)^{m-j}\right)_{1 \leq i, j \leq m}}=\frac{1}{t_{1} \cdots t_{m}} s_{\lambda}^{\mathcal{A}}\left(t_{1}^{2}, \ldots, t_{m}^{2}\right)
\end{aligned}
$$

We remark that $s_{\lambda}^{\mathcal{D}}$ is a symmetric polynomial if $\lambda_{m} \geq 1$. Now the degrees of these Laurent polynomials are following:

$$
\operatorname{deg} s_{\lambda}^{\mathcal{A}}=|\lambda|, \quad \operatorname{deg} s_{\lambda}^{\mathcal{B}}=2|\lambda|+m, \quad \operatorname{deg} s_{\lambda}^{\mathcal{D}}=2|\lambda|-m,
$$

where $|\lambda|:=\lambda_{1}+\cdots+\lambda_{m}$. 
Proposition 3.2. We have the following determinant identities:

$$
\begin{aligned}
\operatorname{det}\left(s_{\lambda}^{\mathcal{A}}\left(x_{\mu}\right)\right)_{\substack{\lambda \in \Lambda \\
\mu \in Z}} & =\left[\prod_{1 \leq i<j \leq \ell}\left(x_{i}-x_{j}\right)\right]^{\left(\begin{array}{c}
\ell-2 \\
m-1
\end{array}\right)}, \\
\operatorname{det}\left(s_{\lambda}^{\mathcal{B}}\left(x_{\mu}\right)\right)_{\substack{\lambda \in \Lambda \\
\mu \in Z}} & =\left(x_{1} \cdots x_{\ell}\right)^{\left(\begin{array}{c}
\ell-1 \\
m-1
\end{array}\right)}\left[\prod_{1 \leq i<j \leq \ell}\left(x_{i}^{2}-x_{j}^{2}\right)\right]^{\left(\begin{array}{c}
\ell-2 \\
m-1
\end{array}\right)}, \\
\operatorname{det}\left(s_{\lambda}^{\mathcal{D}}\left(x_{\mu}\right)\right)_{\substack{\lambda \in \Lambda \\
\mu \in Z}} & =\frac{1}{\left(x_{1} \cdots x_{\ell}\right)^{\left(\begin{array}{l}
\ell-1 \\
m-1
\end{array}\right)}}\left[\prod_{1 \leq i<j \leq \ell}\left(x_{i}^{2}-x_{j}^{2}\right)\right]^{\left(\begin{array}{c}
\ell-2 \\
m-1
\end{array}\right)}
\end{aligned}
$$

Proof. Apply the formula (3.1) to the matrices $A=\left(x_{i}^{\ell-j}\right)_{1 \leq i, j \leq \ell}, A=\left(x_{i}^{2(\ell-j)+1}\right)_{1 \leq i, j \leq \ell}$ and $A=\left(x_{i}^{2(\ell-j)-1}\right)_{1 \leq i, j \leq \ell}$.

We will use these determinant identities for proving that $D^{(2)}(\mathscr{A})$ are free when $\mathscr{A}$ are reflection arrangements types A, B and D in Section 4 and 5 .

Example 3.3. We assume that $\ell=3, m=2$. Then

$$
\Lambda=\left\{\left(\lambda_{1}, \lambda_{2}\right) \mid 1 \geq \lambda_{1} \geq \lambda_{2} \geq 0\right\}=\{(1,1),(1,0),(0,0)\}
$$

The Schur polynomials are following:

$$
\begin{aligned}
& s_{(1,1)}^{\mathcal{A}}\left(t_{1}, t_{2}\right)=\frac{t_{1}^{2} t_{2}-t_{1} t_{2}^{2}}{t_{1}-t_{2}}=t_{1} t_{2}, \\
& s_{(1,0)}^{\mathcal{A}}\left(t_{1}, t_{2}\right)=\frac{t_{1}^{2}-t_{2}^{2}}{t_{1}-t_{2}}=t_{1}+t_{2}, \\
& s_{(0,0)}^{\mathcal{A}}\left(t_{1}, t_{2}\right)=\frac{t_{1}-t_{2}}{t_{1}-t_{2}}=1 .
\end{aligned}
$$

Let

$$
A=\left[\begin{array}{lll}
x_{1}^{2} & x_{1} & 1 \\
x_{2}^{2} & x_{2} & 1 \\
x_{3}^{2} & x_{3} & 1
\end{array}\right]
$$

Then the determinant of $A$ is Vandermonde's determinant, and is equal to the direct product $\left(x_{1}-x_{2}\right)\left(x_{1}-x_{3}\right)\left(x_{2}-x_{3}\right)$. 
Let us consider the second compound matrix $A^{(2)}$ :

$$
\begin{aligned}
A^{(2)}= & {\left[\begin{array}{lll}
x_{1}^{2} x_{2}-x_{1} x_{2}^{2} & x_{1}^{2}-x_{2}^{2} & x_{1}-x_{2} \\
x_{1}^{2} x_{3}-x_{1} x_{3}^{2} & x_{1}^{2}-x_{3}^{2} & x_{1}-x_{3} \\
x_{2}^{2} x_{3}-x_{2} x_{3}^{2} & x_{2}^{2}-x_{3}^{2} & x_{2}-x_{3}
\end{array}\right] } \\
= & {\left[\begin{array}{lll}
\left(x_{1}-x_{2}\right) s_{(1,1)}^{\mathcal{A}}\left(x_{1}, x_{2}\right) & \left(x_{1}-x_{2}\right) s_{(1,0)}^{\mathcal{A}}\left(x_{1}, x_{2}\right) & \left(x_{1}-x_{2}\right) s_{(0,0)}^{\mathcal{A}}\left(x_{1}, x_{2}\right) \\
\left(x_{1}-x_{3}\right) s_{(1,1)}^{\mathcal{A}}\left(x_{1}, x_{3}\right) & \left(x_{1}-x_{3}\right) s_{(1,0)}^{\mathcal{A}}\left(x_{1}, x_{3}\right) & \left(x_{1}-x_{3}\right) s_{(0,0)}^{\mathcal{A}}\left(x_{1}, x_{3}\right) \\
\left(x_{2}-x_{3}\right) s_{(1,1)}^{\mathcal{A}}\left(x_{2}, x_{3}\right) & \left(x_{2}-x_{3}\right) s_{(1,0)}^{\mathcal{A}}\left(x_{2}, x_{3}\right) & \left(x_{2}-x_{3}\right) s_{(0,0)}^{\mathcal{A}}\left(x_{2}, x_{3}\right)
\end{array}\right] . }
\end{aligned}
$$

By the identity (3.1), we have the determinant identity

$$
\left|\begin{array}{lll}
s_{(1,1)}^{\mathcal{A}}\left(x_{1}, x_{2}\right) & s_{(1,0)}^{\mathcal{A}}\left(x_{1}, x_{2}\right) & s_{(0,0)}^{\mathcal{A}}\left(x_{1}, x_{2}\right) \\
s_{(1,1)}^{\mathcal{A}}\left(x_{1}, x_{3}\right) & s_{(1,0)}^{\mathcal{A}}\left(x_{1}, x_{3}\right) & s_{(0,0)}^{\mathcal{A}}\left(x_{1}, x_{3}\right) \\
s_{(1,1)}^{\mathcal{A}}\left(x_{2}, x_{3}\right) & s_{(1,0)}^{\mathcal{A}}\left(x_{2}, x_{3}\right) & s_{(0,0)}^{\mathcal{A}}\left(x_{2}, x_{3}\right)
\end{array}\right|=\left(x_{1}-x_{2}\right)\left(x_{1}-x_{3}\right)\left(x_{2}-x_{3}\right) .
$$

Example 3.4. Let $\ell=3, m=2$. Then

$$
s_{(1,1)}^{\mathcal{B}}\left(t_{1}, t_{2}\right)=\frac{t_{1}^{5} t_{2}^{3}-t_{1}^{3} t_{2}^{5}}{t_{1}^{2}-t_{2}^{2}}, s_{(1,0)}^{\mathcal{B}}\left(t_{1}, t_{2}\right)=\frac{t_{1}^{5} t_{2}-t_{1} t_{2}^{5}}{t_{1}^{2}-t_{2}^{2}}, s_{(0,0)}^{\mathcal{B}}\left(t_{1}, t_{2}\right)=\frac{t_{1}^{3} t_{2}-t_{1} t_{2}^{3}}{t_{1}^{2}-t_{2}^{2}} .
$$

Let

$$
B=\left[\begin{array}{lll}
x_{1}^{5} & x_{1}^{3} & x_{1} \\
x_{2}^{5} & x_{2}^{3} & x_{2} \\
x_{3}^{5} & x_{3}^{3} & x_{3}
\end{array}\right]
$$

Then the determinant $\operatorname{det} B$ is equal to $x_{1} x_{2} x_{3}\left(x_{1}^{2}-x_{2}^{2}\right)\left(x_{1}^{2}-x_{3}^{2}\right)\left(x_{2}^{2}-x_{3}^{2}\right)$. Since $\operatorname{det} B^{(2)}=\left(x_{1} x_{2} x_{3}\left(x_{1}^{2}-x_{2}^{2}\right)\left(x_{1}^{2}-x_{3}^{2}\right)\left(x_{2}^{2}-x_{3}^{2}\right)\right)^{2}$, we have

$$
\left|\begin{array}{lll}
s_{(1,1)}^{\mathcal{B}}\left(x_{1}, x_{2}\right) & s_{(1,0)}^{\mathcal{B}}\left(x_{1}, x_{2}\right) & s_{(0,0)}^{\mathcal{B}}\left(x_{1}, x_{2}\right) \\
s_{(1,1)}^{\mathcal{B}}\left(x_{1}, x_{3}\right) & s_{(1,0)}^{\mathcal{B}}\left(x_{1}, x_{3}\right) & s_{(0,0)}^{\mathcal{B}}\left(x_{1}, x_{3}\right) \\
s_{(1,1)}^{\mathcal{B}}\left(x_{2}, x_{3}\right) & s_{(1,0)}^{\mathcal{B}}\left(x_{2}, x_{3}\right) & s_{(0,0)}^{\mathcal{B}}\left(x_{2}, x_{3}\right)
\end{array}\right|=x_{1}^{2} x_{2}^{2} x_{3}^{2}\left(x_{1}^{2}-x_{2}^{2}\right)\left(x_{1}^{2}-x_{3}^{2}\right)\left(x_{2}^{2}-x_{3}^{2}\right)
$$

\section{Type $A$ and $B$}

Let $\mathscr{A}$ be an arbitrary arrangement. By [3, Proposition 2.3] and [3, Theorem 2.4], we have

$$
D^{(m)}(\mathscr{A})=\bigcap_{H \in \mathscr{A}} D^{(m)}\left(p_{H} S\right)
$$


where $D^{(m)}\left(p_{H} S\right)=\left\{\theta \in D^{(m)}(S) \mid \theta\left(p_{H} x^{\boldsymbol{\alpha}}\right) \in p_{H} S\right.$ for any $\left.|\boldsymbol{\alpha}|=m-1\right\}$ for $H \in$ $\mathscr{A}$.

Recall that the defining polynomials of Coxeter arrangements $\mathcal{A}_{\ell-1}$ and $\mathcal{B}_{\ell}$ of types $A$ and $B$ are

$$
\begin{aligned}
& Q\left(\mathcal{A}_{\ell-1}\right)=\prod_{1 \leq i<j \leq \ell}\left(x_{i}-x_{j}\right), \\
& Q\left(\mathcal{B}_{\ell}\right)=x_{1} \cdots x_{\ell} \prod_{1 \leq i<j \leq \ell}\left(x_{i}^{2}-x_{j}^{2}\right) .
\end{aligned}
$$

We introduce some operators which are in $D^{(m)}\left(\mathcal{A}_{\ell-1}\right)$ or $D^{(m)}\left(\mathcal{B}_{\ell}\right)$. By using these operators, we construct bases for the modules $D^{(2)}\left(\mathcal{A}_{\ell-1}\right)$ and $D^{(2)}\left(\mathcal{B}_{\ell}\right)$ of differential operators of order 2 on $\mathcal{A}_{\ell-1}$ and $\mathcal{B}_{\ell}$.

Let $k=1, \ldots, \ell$, and put $h_{k}^{\mathcal{A}}:=\left(x_{k}-x_{1}\right) \cdots\left(x_{k}-x_{k-1}\right)\left(x_{k}-x_{k+1}\right) \cdots\left(x_{k}-x_{\ell}\right)$ and $h_{k}^{\mathcal{B}}:=x_{k}\left(x_{k}^{2}-x_{1}^{2}\right) \cdots\left(x_{k}^{2}-x_{k-1}^{2}\right)\left(x_{k}^{2}-x_{k+1}^{2}\right) \cdots\left(x_{k}^{2}-x_{\ell}^{2}\right)$. We define operators $\eta_{k}^{\mathcal{A}}$ and $\eta_{k}^{\mathcal{B}}$ in $D^{(m)}(S)$ as follows:

$$
\eta_{k}^{\mathcal{A}}:=h_{k}^{\mathcal{A}} \frac{1}{m !} \partial_{k}^{m}, \quad \eta_{k}^{\mathcal{B}}:=h_{k}^{\mathcal{B}} \frac{1}{m !} \partial_{k}^{m} .
$$

Then $\operatorname{deg} \eta_{k}^{\mathcal{A}}=\ell-1$ and $\operatorname{deg} \eta_{k}^{\mathcal{B}}=2 \ell-1$.

It is convenient to write $f \doteq g$ for $f, g \in S$ if $f=c g$ for some $c \in \mathbb{R}^{\times}$.

Proposition 4.1. For $k=1, \ldots, \ell$, we have that $\eta_{k}^{\mathcal{A}} \in D^{(m)}\left(\mathcal{A}_{\ell-1}\right)$ and $\eta_{k}^{\mathcal{B}} \in$ $D^{(m)}\left(\mathcal{B}_{\ell}\right)$.

Proof. For any $1 \leq i<j \leq \ell$ and a multi-index $\boldsymbol{\beta}$ with $|\boldsymbol{\beta}|=m-1$,

$$
\frac{1}{m !} \partial_{k}^{m}\left(\left(x_{i} \pm x_{j}\right) x^{\boldsymbol{\beta}}\right)= \begin{cases}1 & \text { if } i=k \text { and } \beta_{i}+1=m \\ \pm 1 & \text { if } j=k \text { and } \beta_{j}+1=m \\ 0 & \text { otherwise }\end{cases}
$$

If $i=k$ and $\beta_{i}+1=m$ or $j=k$ and $\beta_{i}+1=m$, then $\eta_{k}^{\mathcal{A}}\left(\left(x_{i}-x_{j}\right) \cdot x^{\boldsymbol{\beta}}\right) \doteq h_{k}^{\mathcal{A}} \in$ $\left(x_{i}-x_{j}\right) S$. Therefore we obtain $\eta_{k}^{\mathcal{A}} \in D^{(m)}\left(\mathcal{A}_{\ell-1}\right)$ from (4.1).

Similarly we have $\eta_{k}^{\mathcal{B}} \in \bigcap_{1 \leq i<j \leq \ell} D^{(m)}\left(\left(x_{i}^{2}-x_{j}^{2}\right) S\right)$. For $i=1, \ldots, \ell$ and a multiindex $\boldsymbol{\beta}$ with $|\boldsymbol{\beta}|=m-1$, we have

$$
\eta_{k}^{\mathcal{B}}\left(x_{i} \cdot x^{\boldsymbol{\beta}}\right)= \begin{cases}h_{k}^{\mathcal{B}} & \text { if } i=k \text { and } \beta_{i}+1=m \\ 0 & \text { otherwise }\end{cases}
$$

This leads to that $\eta_{k}^{\mathcal{B}} \in \bigcap_{i=1}^{\ell} D^{(m)}\left(x_{i} S\right)$. Therefore we obtain $\eta_{k}^{\mathcal{B}} \in D^{(m)}\left(\mathcal{B}_{\ell}\right)$. 
For a Laurent polynomial $f\left(t_{1}, \ldots, t_{m}\right) \in S\left[t_{1}^{ \pm 1}, \ldots, t_{m}^{ \pm 1}\right]$ satisfying $f\left(x_{\boldsymbol{\alpha}}\right) \in S$ for any $\boldsymbol{\alpha}$ with $|\boldsymbol{\alpha}|=m$, we define an operator

$$
\theta_{f}:=\sum_{|\boldsymbol{\alpha}|=m} f\left(x_{\boldsymbol{\alpha}}\right) \frac{1}{\boldsymbol{\alpha} !} \partial^{\boldsymbol{\alpha}} .
$$

We say a Laurent polynomial $f\left(t_{1}, \ldots, t_{m}\right)$ is symmetric if

$$
f\left(t_{1}, \ldots, t_{i}, \ldots, t_{j}, \ldots, t_{m}\right)=f\left(t_{1}, \ldots, t_{j}, \ldots, t_{i}, \ldots, t_{m}\right)
$$

for all pairs $(i, j)$.

Lemma 4.2. Assume that $f\left(t_{1}, \ldots, t_{m}\right)$ is a symmetric Laurent polynomial. Then we have that $\theta_{f} \in D^{(m)}\left(\mathcal{A}_{\ell-1}\right)$.

Proof. Since $f\left(t_{1}, \ldots, t_{m}\right)$ is symmetric, we have

$$
\left.\theta_{f}\left(\left(x_{i}-x_{j}\right) \cdot x^{\boldsymbol{\beta}}\right)\right|_{x_{i}=x_{j}}=\left.\left(f\left(x_{\boldsymbol{\beta}+\boldsymbol{e}_{i}}\right)-f\left(x_{\boldsymbol{\beta}+\boldsymbol{e}_{j}}\right)\right)\right|_{x_{i}=x_{j}}=0
$$

for any $1 \leq i<j \leq \ell$ and a multi-index $\boldsymbol{\beta}$ with $|\boldsymbol{\beta}|=m-1$. We obtain $\theta_{f}\left(\left(x_{i}-x_{j}\right) \cdot x^{\boldsymbol{\beta}}\right) \in\left(x_{i}-x_{j}\right) S$. Hence it follows from (4.1) that $\theta_{f} \in D^{(m)}\left(\mathcal{A}_{\ell-1}\right)$.

For $\lambda \in \Lambda$, define operators

$$
\theta_{\lambda}^{\mathcal{A}}:=\sum_{|\boldsymbol{\alpha}|=m} s_{\lambda}^{\mathcal{A}}\left(x_{\boldsymbol{\alpha}}\right) \frac{1}{\boldsymbol{\alpha} !} \partial^{\boldsymbol{\alpha}}, \quad \theta_{\lambda}^{\mathcal{B}}:=\sum_{|\boldsymbol{\alpha}|=m} s_{\lambda}^{\mathcal{B}}\left(x_{\boldsymbol{\alpha}}\right) \frac{1}{\boldsymbol{\alpha} !} \partial^{\boldsymbol{\alpha}} .
$$

Then $\operatorname{deg} \theta_{\lambda}^{\mathcal{A}}=|\lambda|, \operatorname{deg} \theta_{\lambda}^{\mathcal{B}}=2|\lambda|+m$ by the formula (3.7).

Proposition 4.3. For $\lambda \in \Lambda$, we have $\theta_{\lambda}^{\mathcal{A}} \in D^{(m)}\left(\mathcal{A}_{\ell-1}\right)$ and $\theta_{\lambda}^{\mathcal{B}} \in D^{(m)}\left(\mathcal{B}_{\ell}\right)$.

Proof. Since Laurent polynomials $s_{\lambda}^{\mathcal{A}}$ and $s_{\lambda}^{\mathcal{B}}$ are symmetric, we obtain $\theta_{\lambda}^{\mathcal{A}}, \theta_{\lambda}^{\mathcal{B}} \in$ $D^{(m)}\left(\mathcal{A}_{\ell-1}\right)$ by Lemma 4.2.

By (4.1), we can write

$$
D^{(m)}\left(\mathcal{B}_{\ell}\right)=D^{(m)}\left(\mathcal{A}_{\ell-1}\right) \cap\left(\bigcap_{i=1}^{\ell} D^{(m)}\left(x_{i} S\right)\right) \cap\left(\bigcap_{1 \leq i<j \leq \ell} D^{(m)}\left(\left(x_{i}+x_{j}\right) S\right)\right) .
$$

Thus we only need to prove that

$$
\theta_{\lambda}^{\mathcal{B}} \in\left(\bigcap_{i=1}^{\ell} D^{(m)}\left(x_{i} S\right)\right) \quad \text { and } \quad \theta_{\lambda}^{\mathcal{B}} \in\left(\bigcap_{1 \leq i<j \leq \ell} D^{(m)}\left(\left(x_{i}+x_{j}\right) S\right)\right) .
$$


For any $i=1, \ldots, \ell$ and a multi-index $\boldsymbol{\beta}$ with $|\boldsymbol{\beta}|=m-1$, we have

$$
\theta_{\lambda}^{\mathcal{B}}\left(x_{i} \cdot x^{\boldsymbol{\beta}}\right)=s_{\lambda}^{\mathcal{B}}\left(x_{\boldsymbol{\beta}+\boldsymbol{e}_{i}}\right)=x_{i} \cdot x^{\boldsymbol{\beta}} s_{\lambda}^{\mathcal{A}}\left(x_{\boldsymbol{\beta}+\boldsymbol{e}_{i}}^{2}\right) \in x_{i} S .
$$

This implies $\bigcap_{i=1}^{\ell} \theta_{\lambda}^{\mathcal{B}} \in D^{(m)}\left(x_{i} S\right)$.

For any $1 \leq i<j \leq \ell$ and a multi-index $\boldsymbol{\beta}$ with $|\boldsymbol{\beta}|=m-1$,

$$
\theta_{\lambda}^{\mathcal{B}}\left(\left(x_{i}+x_{j}\right) \cdot x^{\boldsymbol{\beta}}\right)=s_{\lambda}^{\mathcal{B}}\left(x_{\boldsymbol{\beta}+\boldsymbol{e}_{i}}\right)+s_{\lambda}^{\mathcal{B}}\left(x_{\boldsymbol{\beta}+\boldsymbol{e}_{j}}\right)=x^{\boldsymbol{\beta}}\left(x_{i} s_{\lambda}^{\mathcal{A}}\left(x_{\boldsymbol{\beta}+\boldsymbol{e}_{i}}^{2}\right)+x_{j} s_{\lambda}^{\mathcal{A}}\left(x_{\boldsymbol{\beta}+\boldsymbol{e}_{j}}^{2}\right)\right)
$$

Then we have $\left.\theta_{\lambda}^{\mathcal{B}}\left(\left(x_{i}+x_{j}\right) \cdot x^{\boldsymbol{\beta}}\right)\right|_{x_{i}=-x_{j}}=0$, and this implies $\theta_{\lambda}^{\mathcal{B}}\left(\left(x_{i}+x_{j}\right) \cdot x^{\boldsymbol{\beta}}\right) \in$ $\left(x_{i}+x_{j}\right) S$. Hence we obtain $\theta_{\lambda}^{\mathcal{B}} \in D^{(m)}\left(\mathcal{B}_{\ell}\right)$.

Theorem 4.4. Let $m=2$.

(1) The set

$$
C_{\mathcal{A}}:=\left\{\eta_{i}^{\mathcal{A}} \mid i=1, \ldots \ell\right\} \cup\left\{\theta_{\lambda}^{\mathcal{A}} \mid \lambda \in \Lambda\right\}
$$

forms an $S$-basis for $D^{(2)}\left(\mathcal{A}_{\ell-1}\right)$. Hence

$$
\exp D^{(2)}\left(\mathcal{A}_{\ell-1}\right)=\{\ell-1, \ldots, \ell-1\} \cup\{|\lambda| \mid \lambda \in \Lambda\} .
$$

(2) The set

$$
C_{\mathcal{B}}:=\left\{\eta_{i}^{\mathcal{B}} \mid i=1, \ldots \ell\right\} \cup\left\{\theta_{\lambda}^{\mathcal{B}} \mid \lambda \in \Lambda\right\}
$$

forms an $S$-basis for $D^{(2)}\left(\mathcal{B}_{\ell}\right)$. Hence

$$
\exp D^{(2)}\left(\mathcal{B}_{\ell}\right)=\{2 \ell-1, \ldots, 2 \ell-1\} \cup\{2|\lambda|+2 \mid \lambda \in \Lambda\} .
$$

Proof. (1) All operators in $C_{\mathcal{A}}$ belong to $D^{(2)}\left(\mathcal{A}_{\ell-1}\right)$ by Proposition 4.1 and Proposition 4.3 .

By Proposition 2.1, we only need to prove that the determinant of the coefficient matrix $M_{m}\left(C_{\mathcal{A}}\right)$ of the operators of $C_{\mathcal{A}}$ is equal to $Q\left(\mathcal{A}_{\ell-1}\right)^{\ell}$ up to a nonzero constant. By Proposition 3.2, we obtain $\operatorname{det}\left(s_{\lambda}^{\mathcal{A}}\left(x_{\boldsymbol{\alpha}}\right)\right)_{\lambda \in \Lambda, \boldsymbol{\alpha} \in Z}=Q(\mathcal{A})^{\ell-2}$. Hence we have $\operatorname{det} M_{m}\left(C_{\mathcal{A}}\right) \doteq Q\left(\mathcal{A}_{\ell-1}\right)^{2}\left|\begin{array}{cc}I_{\ell} & * \\ 0 & \operatorname{det}\left(s_{\lambda}^{\mathcal{A}}\left(x_{\boldsymbol{\alpha}}\right)\right)_{\substack{\boldsymbol{\alpha} \in \Lambda \\ \boldsymbol{\alpha} \in \boldsymbol{Z}}}\end{array}\right|=Q\left(\mathcal{A}_{\ell-1}\right)^{2} \cdot Q\left(\mathcal{A}_{\ell-1}\right)^{\ell-2}=Q\left(\mathcal{A}_{\ell-1}\right)^{\ell}$.

(2) We have an identity

$$
\operatorname{det} M_{m}\left(C_{\mathcal{B}}\right) \doteq x_{1} \cdots x_{\ell}\left(\prod_{1 \leq i<j \leq \ell}\left(x_{i}^{2}-x_{j}^{2}\right)\right)^{2} \mid \begin{array}{cc}
I_{\ell} & { }^{*}{ }^{*} \\
0 & \operatorname{det}\left(s_{\lambda}^{\mathcal{B}}\left(x_{\boldsymbol{\alpha}}\right)\right)_{\substack{\boldsymbol{\alpha} \in \Lambda \\
\boldsymbol{\alpha} \in Z}}
\end{array}=Q\left(\mathcal{B}_{\ell}\right)^{\ell}
$$

by Proposition 3.2. Then the rest of proof for (2) is similar to the one for (1). 
Example 4.5. Let $\ell=3, m=2$. Then we have $s_{2}=\left(\begin{array}{c}3+2-1 \\ 2\end{array}\right)=6$ and $t_{2}=$ $\left(\begin{array}{c}3+2-2 \\ 2-1\end{array}\right)=3$. List the operators of the set $C_{\mathcal{A}}$ :

$$
\begin{aligned}
& \eta_{1}^{\mathcal{A}}=\left(x_{1}-x_{2}\right)\left(x_{1}-x_{3}\right) \frac{1}{2} \partial_{1}^{2}, \\
& \eta_{2}^{\mathcal{A}}=\left(x_{2}-x_{1}\right)\left(x_{2}-x_{3}\right) \frac{1}{2} \partial_{2}^{2}, \\
& \eta_{3}^{\mathcal{A}}=\left(x_{3}-x_{1}\right)\left(x_{3}-x_{2}\right) \frac{1}{2} \partial_{3}^{2}, \\
& \theta_{(1,1)}^{\mathcal{A}}=x_{1}^{2} \frac{1}{2} \partial_{1}^{2}+x_{2}^{2} \frac{1}{2} \partial_{2}^{2}+x_{3}^{2} \frac{1}{2} \partial_{3}^{2}+x_{1} x_{2} \partial_{1} \partial_{2}+x_{1} x_{3} \partial_{1} \partial_{3}+x_{2} x_{3} \partial_{2} \partial_{3}, \\
& \theta_{(1,0)}^{\mathcal{A}}=2 x_{1} \frac{1}{2} \partial_{1}^{2}+2 x_{2} \frac{1}{2} \partial_{2}^{2}+2 x_{3} \frac{1}{2} \partial_{3}^{2}+\left(x_{1}+x_{2}\right) \partial_{1} \partial_{2}+\left(x_{1}+x_{3}\right) \partial_{1} \partial_{3}+\left(x_{2}+x_{3}\right) \partial_{2} \partial_{3}, \\
& \theta_{(0,0)}^{\mathcal{A}}=\frac{1}{2} \partial_{1}^{2}+\frac{1}{2} \partial_{2}^{2}+\frac{1}{2} \partial_{3}^{2}+\partial_{1} \partial_{2}+\partial_{1} \partial_{3}+\partial_{2} \partial_{3} .
\end{aligned}
$$

Hence the determinant of the coefficient matrix of operators above is

$$
\begin{aligned}
& \operatorname{det} M_{2}\left(\eta_{1}^{\mathcal{A}}, \eta_{2}^{\mathcal{A}}, \eta_{3}^{\mathcal{A}}, \theta_{(1,1)}^{\mathcal{A}}, \theta_{(1,0)}^{\mathcal{A}}, \theta_{(0,0)}^{\mathcal{A}}\right) \\
& =\left|\begin{array}{cccccc}
\left(x_{1}-x_{2}\right)\left(x_{1}-x_{3}\right) & 0 & 0 & \frac{1}{2} x_{1}^{2} & x_{1} & \frac{1}{2} \\
0 & \left(x_{2}-x_{1}\right)\left(x_{2}-x_{3}\right) & 0 & \frac{1}{2} x_{2}^{2} & x_{2} & \frac{1}{2} \\
0 & 0 & \left(x_{3}-x_{1}\right)\left(x_{3}-x_{2}\right) & \frac{1}{2} x_{3}^{2} & x_{2} & \frac{1}{2} \\
0 & 0 & 0 & x_{1} x_{2} & x_{1}+x_{2} & 1 \\
0 & 0 & 0 & x_{1} x_{3} & x_{1}+x_{3} & 1 \\
0 & 0 & 0 & x_{2} x_{3} & x_{2}+x_{3} & 1
\end{array}\right| \\
& =-\left(x_{1}-x_{2}\right)^{2}\left(x_{1}-x_{3}\right)^{2}\left(x_{2}-x_{3}\right)^{2}\left|\begin{array}{lll}
x_{1} x_{2} & x_{1}+x_{2} & 1 \\
x_{1} x_{3} & x_{1}+x_{3} & 1 \\
x_{2} x_{3} & x_{2}+x_{3} & 1
\end{array}\right| \\
& \doteq Q\left(\mathcal{A}_{2}\right)^{3} \text {. }
\end{aligned}
$$

\section{Type $D$}

In this section, we assume $m=2$, and we construct a basis for $D^{(2)}\left(\mathcal{D}_{\ell}\right)$. Recall the defining polynomial $Q\left(\mathcal{D}_{\ell}\right)=\prod_{1 \leq i<j \leq \ell}\left(x_{i}^{2}-x_{j}^{2}\right)$ of the Coxeter arrangement of type $D$.

Set

$$
\begin{aligned}
& \Lambda^{\prime}:=\left\{\lambda=\left(\lambda_{1}, \lambda_{2}\right) \mid \ell-2 \geq \lambda_{1} \geq \lambda_{2} \geq 1\right\} \\
& \Lambda^{\prime \prime}:=\left\{\lambda=\left(\lambda_{1}, \lambda_{2}\right) \mid \ell-2 \geq \lambda_{1} \geq 0, \lambda_{2}=0\right\}
\end{aligned}
$$


Then $\Lambda=\Lambda^{\prime} \cup \Lambda^{\prime \prime}$. Put $\lambda^{(0)}:=(0,0)$. We define operators $\theta_{\lambda}^{\mathcal{D}}$ as follows:

$$
\begin{aligned}
& \theta_{\lambda}^{\mathcal{D}}:=\sum_{|\boldsymbol{\alpha}|=2} s_{\lambda}^{\mathcal{D}}\left(x_{\boldsymbol{\alpha}}\right) \frac{1}{\boldsymbol{\alpha} !} \partial^{\boldsymbol{\alpha}} \quad \text { if } \lambda \in \Lambda^{\prime}, \\
& \theta_{\lambda}^{\mathcal{D}}:=\left(x_{1} \cdots x_{\ell}\right) \sum_{|\boldsymbol{\alpha}|=2} s_{\lambda}^{\mathcal{D}}\left(x_{\boldsymbol{\alpha}}\right) \frac{1}{\boldsymbol{\alpha} !} \partial^{\boldsymbol{\alpha}} \quad \text { if } \lambda \in \Lambda^{\prime \prime} \backslash\left\{\lambda^{(0)}\right\} \\
& \theta_{\lambda}^{\mathcal{D}}:=\left(x_{1} \cdots x_{\ell}\right)^{2} \sum_{|\boldsymbol{\alpha}|=2} s_{\lambda}^{\mathcal{D}}\left(x_{\boldsymbol{\alpha}}\right) \frac{1}{\boldsymbol{\alpha} !} \partial^{\boldsymbol{\alpha}} \quad \text { if } \lambda=\lambda^{(0)} .
\end{aligned}
$$

If $\lambda \in \Lambda^{\prime}$, then we have

$$
s_{\lambda}^{\mathcal{D}}=\frac{\operatorname{det}\left(t_{i}^{2\left(\lambda_{j}-1+2-j\right)+1}\right)_{1 \leq i, j \leq 2}}{\operatorname{det}\left(t_{i}^{2(2-j)}\right)_{1 \leq i, j \leq 2}}=s_{\lambda-\mathbf{1}}^{\mathcal{B}},
$$

where $\lambda-\mathbf{1}=\left(\lambda_{1}-1, \lambda_{2}-1\right)$.

If $\lambda \in \Lambda^{\prime \prime} \backslash\left\{\lambda^{(0)}\right\}$, then

$$
s_{\lambda}^{\mathcal{D}}=\frac{t_{1}^{2 \lambda_{1}+1} \cdot t_{2}^{-1}-t_{2}^{2 \lambda_{1}+1} \cdot t_{1}^{-1}}{t_{1}^{2}-t_{2}^{2}}=\frac{1}{t_{1} t_{2}} \sum_{j=0}^{\lambda_{1}} t_{1}^{2 j} t_{2}^{2\left(\lambda_{1}-j\right)} .
$$

Thus $\left(x_{1} \cdots x_{\ell}\right) s_{\lambda}^{\mathcal{D}}\left(x_{\boldsymbol{\alpha}}\right)$ is a polynomial for any multi-index $\boldsymbol{\alpha}$ with $|\boldsymbol{\alpha}|=2$.

We have

$$
\theta_{\lambda^{(0)}}^{\mathcal{D}}=\left(x_{1} \cdots x_{\ell}\right)^{2}\left(\sum_{i=1}^{\ell} \frac{1}{2 x_{i}^{2}} \partial_{i}^{2}+\sum_{1 \leq i<j \leq \ell} \frac{1}{x_{i} x_{j}} \partial_{i} \partial_{j}\right)
$$

Hence $\theta_{\lambda}^{\mathcal{D}}$ for any $\lambda \in \Lambda$. The degrees of these operators are as follows:

$$
\begin{aligned}
& \operatorname{deg} \theta_{\lambda}^{\mathcal{D}}=2|\lambda|-2=2 \lambda_{1}+2 \lambda_{2}-2 \quad \text { if } \quad \lambda \in \Lambda^{\prime}, \\
& \operatorname{deg} \theta_{\lambda}^{\mathcal{D}}=2 \lambda_{1}-2+\ell \quad \text { if } \quad \lambda \in \Lambda^{\prime \prime} \backslash\left\{\lambda^{(0)}\right\}, \\
& \operatorname{deg} \theta_{\lambda}^{\mathcal{D}}=2 \ell-2 \quad \text { if } \quad \lambda=\lambda^{(0)} .
\end{aligned}
$$

Proposition 5.1. For $\lambda \in \Lambda$, we have $\theta_{\lambda}^{\mathcal{D}} \in D^{(2)}\left(\mathcal{D}_{\ell}\right)$.

Proof. By Lemma 4.2, we have $\theta_{\lambda}^{\mathcal{D}} \in D^{(2)}\left(\mathcal{A}_{\ell-1}\right)$ for any $\lambda \in \Lambda$. 
Since

$$
\begin{aligned}
& \left(\sum_{|\boldsymbol{\alpha}|=2} s_{\lambda}^{\mathcal{D}}\left(x_{\boldsymbol{\alpha}}\right) \frac{1}{\boldsymbol{\alpha} !} \partial^{\boldsymbol{\alpha}}\right)\left(\left(x_{i}+x_{j}\right) \cdot x_{k}\right) \\
& =s_{\lambda}^{\mathcal{D}}\left(x_{i}, x_{k}\right)+s_{\lambda}^{\mathcal{D}}\left(x_{j}, x_{k}\right) \\
& =\frac{1}{x_{i} x_{k}} s_{\lambda}^{\mathcal{A}}\left(x_{i}^{2}, x_{k}^{2}\right)+\frac{1}{x_{j} x_{k}} s_{\lambda}^{\mathcal{A}}\left(x_{j}^{2}, x_{k}^{2}\right) \\
& =\frac{1}{x_{i} x_{j} x_{k}}\left(x_{j} s_{\lambda}^{\mathcal{A}}\left(x_{i}^{2}, x_{k}^{2}\right)+x_{i} s_{\lambda}^{\mathcal{A}}\left(x_{j}^{2}, x_{k}^{2}\right)\right),
\end{aligned}
$$

we obtain $\left.\theta_{\lambda}^{\mathcal{D}}\left(\left(x_{i}+x_{j}\right) x_{k}\right)\right|_{x_{i}=-x_{j}}=0$ for $1 \leq i<j \leq \ell, k=1, \ldots, \ell$ and $\lambda \in \Lambda$. Hence we have $\theta_{\lambda}^{\mathcal{D}} \in D^{(2)}(\mathcal{D})$ for any $\lambda \in \Lambda$.

We introduce other operators $h_{k}^{\mathcal{D}}$ of $D^{(2)}\left(\mathcal{D}_{\ell}\right)$. For $k=1, \ldots, \ell$ put $h_{k}^{\mathcal{D}}:=\left(x_{k}^{2}-\right.$ $\left.x_{1}^{2}\right) \cdots\left(x_{k}^{2}-x_{k-1}^{2}\right)\left(x_{k}^{2}-x_{k+1}^{2}\right) \cdots\left(x_{k}^{2}-x_{\ell}^{2}\right)$, and define

$$
\eta_{k}^{\mathcal{D}}:=\frac{h_{k}^{\mathcal{D}}}{2 x_{k}} \partial_{k}^{2}-(-1)^{\ell-1} \frac{1}{x_{k}} \theta_{\lambda^{(0)}}^{\mathcal{D}}
$$

The coefficient of $\partial_{k}^{2}$ in $\eta_{k}^{\mathcal{D}}$ is

$$
\frac{h_{k}^{\mathcal{D}}}{2 x_{k}}-(-1)^{\ell-1} \frac{\left(x_{1} \cdots x_{\ell}\right)^{2}}{2 x_{k} \cdot x_{k}^{2}}=\frac{h_{k}^{\mathcal{D}}-(-1)^{\ell-1}\left(x_{1} \cdots x_{k-1} x_{k+1} \cdots x_{\ell}\right)^{2}}{2 x_{k}} \in S .
$$

Hence we obtain $\eta_{k}^{\mathcal{D}} \in D^{(2)}(S)$, and $\operatorname{deg} \eta_{k}^{\mathcal{D}}=2 \ell-2$.

Proposition 5.2. For $k=1, \ldots, \ell$, we have that $\eta_{k}^{\mathcal{D}} \in D^{(2)}\left(\mathcal{D}_{\ell}\right)$.

Proof. Let $k=1, \ldots, \ell$. It is clear that $\frac{h_{k}^{\mathcal{D}}}{2} \partial_{k}^{2} \in D^{(2)}\left(\mathcal{D}_{\ell}\right)$, and we have $\theta_{\lambda^{(0)}}^{\mathcal{D}} \in$ $D^{(2)}\left(\mathcal{D}_{\ell}\right)$ by Proposition 5.1. Thus we have $\frac{h_{k}^{\mathcal{D}}}{2} \partial_{k}^{2}-(-1)^{\ell-1} \theta_{\lambda^{(0)}}^{\mathcal{D}} \in D^{(2)}\left(\mathcal{D}_{\ell}\right)$. This leads to $\eta_{k}^{\mathcal{D}} \in D^{(2)}\left(\mathcal{D}_{\ell}\right)$.

Theorem 5.3. Assume $m=2$. The set

$$
C_{\mathcal{D}}:=\left\{\eta_{i}^{\mathcal{D}} \mid i=1, \ldots \ell\right\} \cup\left\{\theta_{\lambda}^{\mathcal{D}} \mid \lambda \in \Lambda\right\}
$$

forms an $S$-basis for $D^{(2)}\left(\mathcal{D}_{\ell}\right)$. Hence

$$
\begin{aligned}
\exp D^{(2)}\left(\mathcal{D}_{\ell}\right)= & \{2 \ell-2, \ldots, 2 \ell-2\} \cup\left\{2 \lambda_{1}+2 \lambda_{2}-2 \mid \ell-2 \geq \lambda_{1} \geq \lambda_{2} \geq 1\right\} \\
& \cup\left\{2 \lambda_{1}-2+\ell \mid \ell-2 \geq \lambda_{1} \geq 1\right\} \cup\{2 \ell-2\}
\end{aligned}
$$


Proof. By Proposition 5.1 and Proposition 5.2, we have $C_{\mathcal{D}} \subseteq D^{(2)}\left(\mathcal{D}_{\ell}\right)$. Let $M_{2}\left(C_{\mathcal{D}}\right)$ be the coefficient matrix of the operators in $C_{\mathcal{D}}$. We shall show that $\operatorname{det} M_{2}\left(C_{\mathcal{D}}\right) \doteq$ $Q\left(\mathcal{D}_{\ell}\right)^{\ell}$.

Put $\theta_{\lambda}:=\sum_{|\boldsymbol{\alpha}|=2} s_{\lambda}^{\mathcal{D}}\left(x_{\boldsymbol{\alpha}}\right) \frac{1}{\boldsymbol{\alpha} !} \partial^{\boldsymbol{\alpha}}$ for $\lambda \in \Lambda$. Then

$$
\begin{aligned}
& \operatorname{det} M_{2}\left(C_{\mathcal{D}}\right)=\operatorname{det} M_{2}\left(\eta_{i}^{\mathcal{D}}, \theta_{\lambda}^{\mathcal{D}} \mid i=1, \ldots, \ell, \lambda \in \Lambda\right) \\
& =\operatorname{det} M_{2}\left(\eta_{i}^{\mathcal{D}}+(-1)^{\ell-1} \frac{1}{x_{i}} \theta_{\lambda^{(0)}}^{\mathcal{D}}, \theta_{\lambda}^{\mathcal{D}} \mid i=1, \ldots, \ell, \lambda \in \Lambda\right) \\
& =\left(x_{1} \cdots x_{\ell}\right)^{\ell} \operatorname{det} M_{2}\left(\eta_{i}^{\mathcal{D}}+(-1)^{\ell-1} \frac{1}{x_{i}} \theta_{\lambda^{(0)}}^{\mathcal{D}}, \theta_{\lambda} \mid i=1, \ldots, \ell, \lambda \in \Lambda\right) \\
& \doteq\left(\frac{h_{1}^{\mathcal{D}}}{x_{1}} \cdots \frac{h_{\ell}^{\mathcal{D}}}{x_{\ell}}\right)\left(x_{1} \cdots x_{\ell}\right)^{\ell}\left|\begin{array}{cc}
I_{\ell} & \operatorname{det}\left(s_{\lambda}^{\mathcal{D}}\left(x_{\boldsymbol{\alpha}}\right)\right)_{\substack{\lambda \in \Lambda \\
\boldsymbol{\alpha} \in Z}} \\
0 & \operatorname{den}
\end{array}\right| \\
& =Q\left(\mathcal{D}_{\ell}\right)^{2}\left(x_{1} \cdots x_{\ell}\right)^{\ell-1} \frac{Q\left(\mathcal{D}_{\ell}\right)^{\ell-2}}{\left(x_{1} \cdots x_{\ell}\right)^{\ell-1}}=Q\left(\mathcal{D}_{\ell}\right)^{\ell}
\end{aligned}
$$

by Proposition 3.2. Hence we conclude that the set $C_{\mathcal{D}}$ forms an $S$-basis for $D^{(2)}\left(\mathcal{D}_{\ell}\right)$ by Proposition 2.1 .

\section{Group actions}

Let $W$ be a finite reflection group generated by reflections acting on $V$. Then $W$ acts on $S$ by $(w \cdot f)(v)=f\left(w^{-1} \cdot v\right)$ for $f \in S, w \in W$ and $v \in V$. The action of $W$ on $D^{(m)}(S)$ is defined by $(w \cdot \theta)(f)=w \cdot\left(\theta\left(w^{-1} \cdot f\right)\right)$ for $w \in W, \theta \in D^{(m)}(S)$ and $f \in S$.

Let $\mathfrak{S}_{\ell}$ be the symmetric group acting on $V$ by permuting the coordinates. Let $\mathbb{Z} / 2 \mathbb{Z}=\{1,-1\}$. An abelian group $(\mathbb{Z} / 2 \mathbb{Z})^{\ell}$ acts on $V$ by change of signs. Let $(\mathbb{Z} / 2 \mathbb{Z})^{\ell-1}$ be the subgroup of $(\mathbb{Z} / 2 \mathbb{Z})^{\ell}$ defined by

$$
(\mathbb{Z} / 2 \mathbb{Z})^{\ell-1}=\left\{\left(a_{1}, \ldots, a_{\ell}\right) \in(\mathbb{Z} / 2 \mathbb{Z})^{\ell} \mid a_{1} \cdots a_{\ell}=1\right\}
$$

The group $\mathfrak{S}_{\ell}$ acts on $(\mathbb{Z} / 2 \mathbb{Z})^{\ell}$ and $(\mathbb{Z} / 2 \mathbb{Z})^{\ell-1}$ by permuting the coodinates.

The finite irreducible reflection groups of types A, B and D are defined by

$$
\begin{aligned}
& W^{A}:=\mathfrak{S}_{\ell}, \\
& W^{B}:=\mathfrak{S}_{\ell} \ltimes(\mathbb{Z} / 2 \mathbb{Z})^{\ell}, \\
& W^{D}:=\mathfrak{S}_{\ell} \ltimes(\mathbb{Z} / 2 \mathbb{Z})^{\ell-1} .
\end{aligned}
$$


From now on, we assume $\ell \geq 4$ when we consider the reflection group of type $\mathrm{D}$. Then the groups $W^{A}, W^{B}$ and $W^{D}$ act on $V$. Hence the groups $W^{A}, W^{B}$ and $W^{D}$ act on $S$ and $D^{(m)}(S)$.

Proposition 6.1. Let $W$ be a finite reflection group, and $\mathscr{A}$ the reflection arrangement consisting of all reflection hyperplanes of $W$. Then the submodule $D^{(m)}(\mathscr{A})$ of $D^{(m)}(S)$ is closed under the action of $W$.

Proof. For $w \in W$ and $\theta \in D^{(m)}(\mathscr{A})$, we prove that $w \cdot \theta \in D^{(m)}(\mathscr{A})$.

For $f \in S$, we have

$$
\begin{aligned}
w \cdot \theta(Q f) & =w \cdot\left(\theta\left(w^{-1} \cdot(Q f)\right)\right) \\
& =w \cdot\left(\theta\left(\operatorname{det}\left(w^{-1}\right) Q\left(w^{-1} \cdot f\right)\right)\right) .
\end{aligned}
$$

Since $\operatorname{det}\left(w^{-1}\right) Q\left(w^{-1} \cdot f\right) \in Q S$, we have $\theta\left(\operatorname{det}\left(w^{-1}\right) Q\left(w^{-1} \cdot f\right)\right) \in Q S$. Then there exists $g \in S$ such that $\theta\left(\operatorname{det}\left(w^{-1}\right) Q\left(w^{-1} \cdot f\right)\right)=Q g$. Hence

$$
w \cdot \theta(Q f)=w \cdot(Q g)=(\operatorname{det}(w) Q)(w \cdot g) \in Q S .
$$

By Proposition 6.1, the groups $W^{A}, W^{B}$ and $W^{D}$ act on $D^{(m)}(\mathcal{A}), D^{(m)}(\mathcal{B})$ and $D^{(m)}(\mathcal{D})$, respectively.

In case $m=1$, the modules $D^{(1)}(\mathcal{A}), D^{(1)}(\mathcal{B})$ and $D^{(1)}(\mathcal{D})$ have bases consisting of only invariant elements [10, Theorem 6.60]. In this section, we prove that $D^{(2)}(\mathcal{A})$, $D^{(2)}(\mathcal{B})$ and $D^{(2)}(\mathcal{D})$ cannot have bases consisting of only invariant elements, when $m=2$.

The actions of a transposition $\sigma_{i, j}:=(i j) \in \mathfrak{S}_{\ell}$ on $\left\{x_{1}, \ldots x_{\ell}\right\}$ and $\left\{\partial_{1}, \ldots, \partial_{\ell}\right\}$ are as follows:

$$
\sigma_{i, j} \cdot x_{k}=x_{\sigma_{i, j} \cdot k}, \sigma_{i, j} \cdot \partial_{k}=\partial_{\sigma_{i, j} \cdot k} \quad(k=1, \ldots, \ell) .
$$

The group $\mathfrak{S}_{\ell}$ acts on the set of multi-indices by permuting the coordinates:

$$
\sigma_{i, j} \cdot\left(\alpha_{1}, \ldots, \alpha_{i}, \ldots, \alpha_{j}, \ldots, \alpha_{\ell}\right)=\left(\alpha_{1}, \ldots, \alpha_{j}, \ldots, \alpha_{i}, \ldots, \alpha_{\ell}\right)
$$

The action of $\mathfrak{S}_{\ell}$ preserves the norm of a multi-index. Then $\sigma_{i, j} \cdot x^{\boldsymbol{\alpha}}=x^{\sigma_{i, j} \cdot \boldsymbol{\alpha}}$ for a multi-index $\boldsymbol{\alpha} \in \mathbb{N}^{\ell}$.

Let $\tau_{i} \in(\mathbb{Z} / 2 \mathbb{Z})^{\ell}$ be the element of change of signs of the $i$-th coordinate:

$$
\tau_{i} \cdot x_{k}=a_{i, k} x_{k}, \tau_{i} \cdot \partial_{k}=a_{i, k} \partial_{k} \quad(k=1, \ldots, \ell)
$$

where $a_{i, i}=-1$ and $a_{i, k}=1$ for $k \neq i$. 
Lemma 6.2. Let $\lambda \in \Lambda$.

(1) The operator $\theta_{\lambda}^{\mathcal{A}}$ is $W^{A}$-invariant.

(2) The operator $\theta_{\lambda}^{\mathcal{B}}$ is $W^{B}$-invariant.

(3) The operator $\theta_{\lambda}^{\mathcal{D}}$ is $W^{D}$-invariant when $m=2$.

Proof. (1) Since $W^{A}$ is generated by transpositions $\sigma_{1,2}, \ldots, \sigma_{\ell-1, \ell}$ (see, for example, [1]), it is enough to prove that

$$
\theta_{\lambda}^{\mathcal{A}}=\sum_{|\boldsymbol{\alpha}|=m} s_{\lambda}^{\mathcal{A}}\left(x_{\boldsymbol{\alpha}}\right) \frac{1}{\boldsymbol{\alpha} !} \partial^{\boldsymbol{\alpha}}
$$

is invariant under the actions of transpositions $\sigma_{1,2}, \ldots, \sigma_{\ell-1, \ell}$.

Clearly, we have that $\left(\sigma_{i, i+1} \cdot \boldsymbol{\alpha}\right) !=\boldsymbol{\alpha}$ ! and $\left|\sigma_{i, i+1} \cdot \boldsymbol{\alpha}\right|=|\boldsymbol{\alpha}|$ for a multi-index $\boldsymbol{\alpha}$ with $|\boldsymbol{\alpha}|=m$. Then a transposition $\sigma_{i, i+1}$ is a bijection between the set $\left\{\boldsymbol{\alpha} \in \mathbb{N}^{\ell} \mid\right.$ $|\boldsymbol{\alpha}|=m\}$ and itself. Therefore we have that, for $i=1, \ldots, \ell-1$,

$$
\begin{aligned}
\sigma_{i, i+1} \cdot \theta_{\lambda}^{\mathcal{A}} & =\sum_{\left|\sigma_{i, i+1} \cdot \boldsymbol{\alpha}\right|=m} \sigma_{i, i+1} \cdot\left(s_{\lambda}^{\mathcal{A}}\left(x_{\sigma_{i, i+1} \cdot \boldsymbol{\alpha}}\right)\right) \frac{1}{\left(\sigma_{i, i+1} \cdot \boldsymbol{\alpha}\right) !} \sigma_{i, i+1} \cdot\left(\partial^{\sigma_{i, i+1} \cdot \boldsymbol{\alpha}}\right) \\
& =\sum_{\left|\sigma_{i, i+1} \cdot \boldsymbol{\alpha}\right|=m} s_{\lambda}^{\mathcal{A}}\left(x_{\sigma_{i, i+1} \cdot \sigma_{i, i+1} \cdot \boldsymbol{\alpha}}\right) \frac{1}{\left(\sigma_{i, i+1} \cdot \boldsymbol{\alpha}\right) !} \partial^{\sigma_{i, i+1} \cdot \sigma_{i, i+1} \cdot \boldsymbol{\alpha}} \\
& =\sum_{|\boldsymbol{\alpha}|=m} s_{\lambda}^{\mathcal{A}}\left(x_{\boldsymbol{\alpha}}\right) \frac{1}{\boldsymbol{\alpha} !} \cdot \partial^{\boldsymbol{\alpha}}=\theta_{\lambda}^{\mathcal{A}} .
\end{aligned}
$$

(2) The group $W^{B}$ is generated by $\tau_{\ell}$ and transpositions $\sigma_{1,2}, \ldots, \sigma_{\ell-1, \ell}$ (see [1]). It is enough to prove that $\theta_{\lambda}^{\mathcal{B}}$ is invariant under the actions of the generators.

By the formulas (1.2) and (3.5), we have

$$
s_{\lambda}^{\mathcal{B}}\left(x_{\boldsymbol{\alpha}}\right)=x_{1}^{\alpha_{1}} \cdots x_{\ell}^{\alpha_{\ell}} s_{\lambda}^{\mathcal{A}}\left(x_{\boldsymbol{\alpha}}^{2}\right)
$$

for a multi-index $\boldsymbol{\alpha}$ with $|\boldsymbol{\alpha}|=m$. Then

$$
\begin{aligned}
\sigma_{i, i+1} \cdot s_{\lambda}^{\mathcal{B}}\left(x_{\boldsymbol{\alpha}}\right) & =x_{\sigma_{i, i+1} \cdot 1}^{\alpha_{1}} \cdots x_{\sigma_{i, i+1} \cdot \ell}^{\alpha_{\ell}} s_{\lambda}^{\mathcal{A}}\left(x_{\sigma_{i, i+1} \cdot \boldsymbol{\alpha}}^{2}\right) \\
& =x_{1}^{\alpha_{\sigma_{i, i+1} \cdot 1}} \cdots x_{\ell}^{\alpha_{\sigma_{i, i+1} \cdot \ell}} s_{\lambda}^{\mathcal{A}}\left(x_{\sigma_{i, i+1} \cdot \boldsymbol{\alpha}}^{2}\right)=s_{\lambda}^{\mathcal{B}}\left(x_{\sigma_{i, i+1} \cdot \boldsymbol{\alpha}}\right) .
\end{aligned}
$$

Hence we have that

$$
\sigma_{i, i+1} \cdot \theta_{\lambda}^{\mathcal{B}}=\sum_{|\boldsymbol{\alpha}|=m} s_{\lambda}^{\mathcal{B}}\left(x_{\sigma_{i, i+1} \cdot \boldsymbol{\alpha}}\right) \frac{1}{\left(\sigma_{i, i+1} \cdot \boldsymbol{\alpha}\right) !} \cdot \partial^{\sigma_{i, i+1} \cdot \boldsymbol{\alpha}}=\theta_{\lambda}^{\mathcal{B}} .
$$


It remains to prove that $\theta_{\lambda}^{\mathcal{B}}$ is $\tau_{\ell}$-invariant.

By the formulas (6.1) and (6.2), we have $\tau_{\ell} \cdot \partial^{\alpha}=(-1)^{\alpha_{\ell}} \partial^{\alpha}$ and $\tau_{\ell} \cdot s_{\lambda}^{\mathcal{B}}\left(x_{\boldsymbol{\alpha}}\right)=$ $(-1)^{\alpha} s_{\lambda}^{\mathcal{B}}\left(x_{\boldsymbol{\alpha}}\right)$. Then we have

$$
\tau_{\ell} \cdot \theta_{\lambda}^{\mathcal{B}}=\sum_{|\boldsymbol{\alpha}|=m}(-1)^{\alpha_{\ell}} s_{\lambda}^{\mathcal{B}}\left(x_{\boldsymbol{\alpha}}\right) \frac{1}{\boldsymbol{\alpha} !}(-1)^{\alpha_{\ell}} \partial^{\tau_{\ell} \cdot \boldsymbol{\alpha}}=\theta_{\lambda}^{\mathcal{B}}
$$

Hence the operator $w \cdot \theta_{\lambda}^{\mathcal{B}}$ coincides with $\theta_{\lambda}^{\mathcal{B}}$ for $w \in W^{B}$.

(3) The proof of (3) goes similarly to (2). The set

$$
\left\{\sigma_{1,2}, \ldots, \sigma_{\ell-1, \ell}, \sigma_{\ell-1, \ell} \tau_{\ell-1} \tau_{\ell}\right\}
$$

is a system of generators of $W^{D}$ (see [1]).

The formulas (1.2) and (3.6) imply that

$$
s_{\lambda}^{\mathcal{D}}\left(x_{\boldsymbol{\alpha}}\right)=\frac{1}{x_{1}^{\alpha_{1}} \cdots x_{\ell}^{\alpha_{\ell}}} s_{\lambda}^{\mathcal{A}}\left(x_{\sigma_{i, i+1} \cdot \boldsymbol{\alpha}}^{2}\right) .
$$

Then the formula (6.3) leads to

$$
\sigma_{i, i+1} \cdot s_{\lambda}^{\mathcal{D}}\left(x_{\boldsymbol{\alpha}}\right)=\frac{1}{\sigma_{i, i+1} \cdot\left(x_{1}^{\alpha_{1}} \cdots x_{\ell}^{\alpha_{\ell}}\right)} s_{\lambda}^{\mathcal{A}}\left(x_{\sigma_{i, i+1} \cdot \boldsymbol{\alpha}}^{2}\right)=s_{\lambda}^{\mathcal{D}}\left(x_{\sigma_{i, i+1} \cdot \boldsymbol{\alpha}}\right) .
$$

We obtain $\sigma_{i, i+1} \cdot \theta_{\lambda}^{\mathcal{D}}=\theta_{\lambda}^{\mathcal{D}}$ by a straightforward calculation using the formulas (5.1), (5.2) and (5.3).

In order to verify $\sigma_{\ell-1, \ell} \tau_{\ell-1} \tau_{\ell} \cdot \theta_{\lambda}^{\mathcal{D}}=\theta_{\lambda}^{\mathcal{D}}$, we compute the actions of $\sigma_{1, \ell-1} \tau_{\ell-1} \tau_{\ell}$ on polynomials and differential operators:

$$
\begin{aligned}
\sigma_{\ell-1, \ell} \tau_{\ell-1} \tau_{\ell} \cdot s_{\lambda}^{\mathcal{D}}\left(x_{\boldsymbol{\alpha}}\right) & =(-1)^{\alpha_{\ell-1}+\alpha_{\ell}} \frac{1}{\sigma_{\ell-1, \ell} \cdot\left(x_{1}^{\alpha_{1}} \cdots x_{\ell}^{\alpha_{\ell}}\right)} s_{\lambda}^{\mathcal{A}}\left(x_{\sigma_{\ell-1, \ell} \cdot \boldsymbol{\alpha}}^{2}\right) \\
& =(-1)^{\alpha_{\ell-1}+\alpha_{\ell}} s_{\lambda}^{\mathcal{D}}\left(x_{\boldsymbol{\alpha}}\right), \\
\sigma_{\ell-1, \ell} \tau_{\ell-1} \tau_{\ell} \cdot \partial^{\boldsymbol{\alpha}} & =(-1)^{\alpha_{\ell-1}+\alpha_{\ell}} \partial^{\sigma_{\ell-1, \ell} \cdot \boldsymbol{\alpha}}, \\
\sigma_{\ell-1, \ell} \tau_{\ell-1} \tau_{\ell} \cdot \frac{1}{x_{1} \cdots x_{\ell}} & =\frac{1}{x_{1} \cdots x_{\ell}}, \\
\sigma_{\ell-1, \ell} \tau_{\ell-1} \tau_{\ell} \cdot \frac{1}{\left(x_{1} \cdots x_{\ell}\right)^{2}} & =\frac{1}{\left(x_{1} \cdots x_{\ell}\right)^{2}} .
\end{aligned}
$$

By the case-by-case checking, we can verify that $\sigma_{\ell-1, \ell} \tau_{\ell-1} \tau_{\ell} \cdot \theta_{\lambda}^{\mathcal{D}}$ coincides with $\theta_{\lambda}^{\mathcal{D}}$ for $\lambda \in \Lambda$. Therefore the operator $\theta_{\lambda}^{\mathcal{D}}$ is $W^{D}$-invariant. 
Lemma 6.3. (1) The vector space spanned by $\left\{\eta_{k}^{\mathcal{A}} \mid k=1, \ldots \ell\right\}$ is closed under the action of $W^{A}$. Moreover, the vector space spanned by $\left\{\eta_{k}^{\mathcal{A}} \mid k=1, \ldots \ell\right\}$ is isomorphic to the Euclidean space $V$ as $W^{A}$-modules.

(2) The vector space spanned by $\left\{\eta_{k}^{\mathcal{B}} \mid k=1, \ldots \ell\right\}$ is closed under the action of $W^{B}$. Moreover, the vector space spanned by $\left\{\eta_{k}^{\mathcal{B}} \mid k=1, \ldots \ell\right\}$ is isomorphic to the Euclidean space $V$ as $W^{B}$-modules.

(3) Let $m=2$. The vector space spanned by $\left\{\eta_{k}^{\mathcal{D}} \mid k=1, \ldots \ell\right\}$ is closed under the action of $W^{D}$. Moreover, the vector space spanned by $\left\{\eta_{k}^{\mathcal{D}} \mid k=1, \ldots \ell\right\}$ is isomorphic to the Euclidean space $V$ as $W^{D}$-modules.

Proof. (1) Let $\left\langle\eta_{1}^{\mathcal{A}}, \ldots, \eta_{\ell}^{\mathcal{A}}\right\rangle_{\mathbb{R}}$ be the vector space spanned by $\left\{\eta_{k}^{\mathcal{D}} \mid k=1, \ldots \ell\right\}$. Define a linear isomorphism

$$
\phi_{A}:\left\langle\eta_{1}^{\mathcal{A}}, \ldots, \eta_{\ell}^{\mathcal{A}}\right\rangle_{\mathbb{R}} \longrightarrow V
$$

by $\phi_{A}\left(\eta_{k}^{\mathcal{A}}\right)=e_{k}$ for $k=1, \ldots, \ell$.

A transposition $\sigma_{i, i+1}$ acts on the standard basis $e_{1}, \ldots, e_{\ell}$ of $V$ by $\sigma_{i, i+1} \cdot e_{k}=$ $e_{\sigma_{i, i+1} \cdot k}$ for $k=1, \ldots, \ell$. To prove the assertion, we verify $\sigma_{i, i+1} \cdot \eta_{k}^{\mathcal{A}}=\eta_{\sigma_{i, i+1} \cdot k}^{\mathcal{A}}$ for $k=1, \ldots, \ell$.

Recall that the definitions $h_{k}^{\mathcal{A}}=\left(x_{k}-x_{1}\right) \cdots\left(x_{k}-x_{k-1}\right)\left(x_{k}-x_{k+1}\right) \cdots\left(x_{k}-x_{\ell}\right)$ and $\eta_{k}^{\mathcal{A}}=h_{k}^{\mathcal{A}} \frac{1}{m !} \partial_{k}^{m}$. For $k \neq i, i+1$, we have $\sigma_{i, i+1} \cdot h_{k}^{\mathcal{A}}=h_{k}^{\mathcal{A}}$. Also we have

$\sigma_{i, i+1} \cdot h_{i}^{\mathcal{A}}=\left(x_{i+1}-x_{1}\right) \cdots\left(x_{i+1}-x_{i-1}\right)\left(x_{i+1}-x_{i}\right)\left(x_{i+1}-x_{i+2}\right) \cdots\left(x_{k}-x_{\ell}\right)=h_{i+1}^{\mathcal{A}}$,

and similarly $\sigma_{i, i+1} \cdot h_{i+1}^{\mathcal{A}}=h_{i}^{\mathcal{A}}$. Then we obtain

$$
\sigma_{i, i+1} \cdot \eta_{k}^{\mathcal{A}}=\eta_{k}^{\mathcal{A}}(k \neq i, i+1), \sigma_{i, i+1} \cdot \eta_{i}^{\mathcal{A}}=\eta_{i+1}^{\mathcal{A}}, \sigma_{i, i+1} \cdot \eta_{i+1}^{\mathcal{A}}=\eta_{i}^{\mathcal{A}}
$$

Hence we conclude that the map $\phi_{A}$ is a $W^{A}$-isomorphism.

(2) A system of generators $\sigma_{1,2}, \ldots, \sigma_{\ell-1, \ell}$ and $\tau_{\ell}$ of $W^{B}$ acts on the standard basis $e_{1}, \ldots, e_{\ell}$ of $V$ by $\sigma_{i, i+1} \cdot e_{k}=e_{\sigma_{i, i+1} \cdot k}$ for $k=1, \ldots, \ell$ and

$$
\tau_{\ell} \cdot e_{\ell}=-e_{\ell}, \tau_{\ell} \cdot e_{k}=e_{k}(k=1, \ldots, \ell-1) .
$$

Define a linear isomorphism

$$
\phi_{B}:\left\langle\eta_{1}^{\mathcal{B}}, \ldots, \eta_{\ell}^{\mathcal{B}}\right\rangle_{\mathbb{R}} \longrightarrow V
$$

by $\phi_{B}\left(\eta_{k}^{\mathcal{B}}\right)=e_{k}$ for $k=1, \ldots, \ell$. We prove that $\phi_{B}$ is a $W^{B}$-isomorphism by checking all actions of the generators are the same. 
Recall that the definitions $h_{k}^{\mathcal{B}}=x_{k}\left(x_{k}^{2}-x_{1}^{2}\right) \cdots\left(x_{k}^{2}-x_{k-1}^{2}\right)\left(x_{k}^{2}-x_{k+1}^{2}\right) \cdots\left(x_{k}^{2}-x_{\ell}^{2}\right)$ and $\eta_{k}^{\mathcal{B}}=h_{k}^{\mathcal{B}} \frac{1}{m !} \partial_{k}^{m}$. We have the following:

$$
\begin{aligned}
\sigma_{i, i+1} \cdot h_{k}^{\mathcal{B}} & =h_{k}^{\mathcal{B}} \quad(k \neq i, i+1), \\
\sigma_{i, i+1} \cdot h_{i}^{\mathcal{B}} & =h_{i+1}^{\mathcal{B}}, \\
\sigma_{i, i+1} \cdot h_{i+1}^{\mathcal{B}} & =h_{i}^{\mathcal{B}}, \\
\tau_{\ell} \cdot h_{k}^{\mathcal{B}} & =h_{k}^{\mathcal{B}} \quad(k \neq \ell), \\
\tau_{\ell} \cdot h_{\ell}^{\mathcal{B}} & =-h_{\ell}^{\mathcal{B}} .
\end{aligned}
$$

Then

$$
\begin{gathered}
\sigma_{i, i+1} \cdot \eta_{k}^{\mathcal{B}}=\eta_{k}^{\mathcal{B}}(k \neq i, i+1), \sigma_{i, i+1} \cdot \eta_{i}^{\mathcal{B}}=\eta_{i+1}^{\mathcal{B}}, \sigma_{i, i+1} \cdot \eta_{i+1}^{\mathcal{B}}=\eta_{i}^{\mathcal{B}}, \\
\tau_{\ell} \cdot \eta_{k}^{\mathcal{B}}=\eta_{k}^{\mathcal{B}}(k \neq \ell), \tau_{\ell} \cdot \eta_{\ell}^{\mathcal{B}}=\eta_{\ell}^{\mathcal{B}} .
\end{gathered}
$$

The actions of the generators on $\left\{\eta_{k}^{\mathcal{B}} \mid k=1, \ldots, \ell\right\}$ coincide with the actions of the generators on $\left\{e_{k} \mid k=1, \ldots, \ell\right\}$. Hence the map $\phi_{B}$ is a $W^{B}$-isomorphism.

(3) The group $W^{D}$ is generated by elements $\sigma_{1,2}, \ldots, \sigma_{\ell-1, \ell}$ and $\sigma_{\ell-1, \ell} \tau_{\ell-1} \tau_{\ell}$. The generators acts on the standard basis $e_{1}, \ldots, e_{\ell}$ of $V$ by $\sigma_{i, i+1} \cdot e_{k}=e_{\sigma_{i, i+1} \cdot k}$ for $k=1, \ldots, \ell$ and

$$
\begin{gathered}
\sigma_{\ell-1, \ell} \tau_{\ell-1} \tau_{\ell} \cdot e_{\ell}=-e_{\ell-1}, \quad \sigma_{\ell-1, \ell} \tau_{\ell-1} \tau_{\ell} \cdot e_{\ell-1}=-e_{\ell}, \\
\sigma_{\ell-1, \ell} \tau_{\ell-1} \tau_{\ell} \cdot e_{k}=e_{k} \quad(k=1, \ldots, \ell-2) .
\end{gathered}
$$

We prove that a linear isomorphism

$$
\phi_{D}:\left\langle\eta_{1}^{\mathcal{D}}, \ldots, \eta_{\ell}^{\mathcal{D}}\right\rangle_{\mathbb{R}} \longrightarrow V
$$

defined by $\phi_{D}\left(\eta_{k}^{\mathcal{D}}\right)=e_{k}$ for $k=1, \ldots, \ell$ is a $W^{D}$-isomorphism.

Recall that the definitions $h_{k}^{\mathcal{D}}=\left(x_{k}^{2}-x_{1}^{2}\right) \cdots\left(x_{k}^{2}-x_{k-1}^{2}\right)\left(x_{k}^{2}-x_{k+1}^{2}\right) \cdots\left(x_{k}^{2}-x_{\ell}^{2}\right)$ and $\eta_{k}^{\mathcal{D}}=\frac{h_{k}^{\mathcal{D}}}{2 x_{k}} \partial_{k}^{2}-(-1)^{\ell-1} \frac{1}{x_{k}} \theta_{\lambda(0)}^{\mathcal{D}}$. Clearly we have

$$
\sigma_{i, i+1} \cdot h_{i}^{\mathcal{D}}=h_{i+1}^{\mathcal{D}}, \sigma_{i, i+1} \cdot h_{i+1}^{\mathcal{D}}=h_{i}^{\mathcal{D}}, \sigma_{i, i+1} \cdot h_{k}^{\mathcal{D}}=h_{k}^{\mathcal{D}}(k \neq i, i+1),
$$

and then

$$
\sigma_{i, i+1} \cdot \eta_{i}^{\mathcal{D}}=\eta_{i+1}^{\mathcal{D}}, \sigma_{i, i+1} \cdot \eta_{i+1}^{\mathcal{D}}=\eta_{i}^{\mathcal{D}}, \sigma_{i, i+1} \cdot \eta_{k}^{\mathcal{D}}=\eta_{k}^{\mathcal{D}}(k \neq i, i+1)
$$

by Lemma 6.2. The actions of $\sigma_{\ell-1, \ell} \tau_{\ell-1} \tau_{\ell}$ on $\left\{h_{k}^{\mathcal{D}} \mid k=1, \ldots, \ell\right\}$ are

$$
\begin{array}{r}
\sigma_{\ell-1, \ell} \tau_{\ell-1} \tau_{\ell} \cdot h_{\ell}^{\mathcal{D}}=h_{\ell-1}^{\mathcal{D}}, \sigma_{\ell-1, \ell} \tau_{\ell-1} \tau_{\ell} \cdot h_{\ell-1}^{\mathcal{D}}=h_{\ell}^{\mathcal{D}}, \\
\sigma_{\ell-1, \ell} \tau_{\ell-1} \tau_{\ell} \cdot h_{k}^{\mathcal{D}}=h_{k}^{\mathcal{D}} \quad(k=1, \ldots, \ell-2) .
\end{array}
$$


Then it follows from Lemma 6.2 that

$$
\begin{aligned}
\sigma_{\ell-1, \ell} \tau_{\ell-1} \tau_{\ell} \cdot \eta_{\ell}^{\mathcal{D}} & =-\frac{h_{\ell-1}^{\mathcal{D}}}{2 x_{\ell-1}} \partial_{\ell-1}^{2}+(-1)^{\ell-1} \frac{1}{x_{\ell-1}} \theta_{\lambda^{(0)}}^{\mathcal{D}}=-\eta_{\ell-1}^{\mathcal{D}}, \\
\sigma_{\ell-1, \ell} \tau_{\ell-1} \tau_{\ell} \cdot \eta_{\ell-1}^{\mathcal{D}} & =-\frac{h_{\ell}^{\mathcal{D}}}{2 x_{\ell}} \partial_{\ell}^{2}+(-1)^{\ell-1} \frac{1}{x_{\ell}} \theta_{\lambda^{(0)}}^{\mathcal{D}}=-\eta_{\ell}^{\mathcal{D}}, \\
\sigma_{\ell-1, \ell} \tau_{\ell-1} \tau_{\ell} \cdot \eta_{k}^{\mathcal{D}} & =\eta_{k}^{\mathcal{D}}(k=1, \ldots, \ell-2) .
\end{aligned}
$$

Thus the actions of the generators on $\left\{\eta_{k}^{\mathcal{D}} \mid k=1, \ldots, \ell\right\}$ coincide with the actions of the generators on $\left\{e_{k} \mid k=1, \ldots, \ell\right\}$. Hence the map $\phi_{D}$ is a $W^{D}$-isomorphism.

Corollary 6.4. Assume that $m=2$. Let $W$ be a finite reflection group of type $A, B$ or $D$. Let $\mathscr{A}$ be the reflection arrangement corresponding to $W$. Then the vector space $X$ generated by the basis $C_{\mathscr{A}}$ in Theorem 4.4 or Theorem 5.3 is a $W$ module, and $X$ is isomorphic as a representeation to $X_{0}^{\sharp \Lambda} \oplus V$ where $X_{0}$ is the trivial representation.

In the case of type $A_{\ell-1}$, the reflection group $W^{A}$ stabilizes the subspace $\mathbb{R}\left(e_{1}+\right.$ $\left.\cdots+e_{\ell}\right)$ of $V$ pointwisely. The orthogonal complement $V^{\prime}$ of $\mathbb{R}\left(e_{1}+\cdots+e_{\ell}\right)$ is closed under the action of the reflection group $W^{A}$, and $V^{\prime}$ is essensial (see [4]). Moreover $V^{\prime}$ is an irreducible representation. In the case of type $\mathrm{B}$ or $\mathrm{D}$, a representation $V$ is irreducible (see Bourbaki [1, Chap. 5, Sect. 3, Proposition 5]).

Let $D^{(m)}(S)^{W}$ be the set of invariant elements of $D^{(m)}(S)$.

Theorem 6.5. Assume that $m=2$. Let $W$ be a finite reflection group of type $A$, $B$ or $D$, and let $\mathscr{A}$ be the reflection arrangement corresponding to $W$. Then The module $D^{(2)}(\mathscr{A})$ cannot have bases consisting of only invariant elements.

Proof. Since proofs of type B and D are almost the same with the proof of type A, we prove the assertion only in the case of type A.

We assume that $\mathscr{A}=\mathcal{A}_{\ell-1}$ and $W=W^{A}$. Let $\theta_{1}^{\prime}, \ldots, \theta_{s 2}^{\prime}$ be an $S$-basis for $D^{(2)}(\mathscr{A})$. Since the degrees do not depend on a choice of a basis, we have $\left\{\operatorname{deg} \theta_{1}^{\prime}, \ldots, \operatorname{deg} \theta_{s_{2}}^{\prime}\right\}=\exp D^{(2)}\left(\mathcal{A}_{\ell-1}\right)$ by Theorem 4.4. Assume $\operatorname{deg} \theta_{1}^{\prime} \leq \cdots \leq$ $\operatorname{deg} \theta_{s_{2}}^{\prime}$. We show that there exists $\theta_{j}^{\prime}$ with $\operatorname{deg} \theta_{j}^{\prime}=\ell-1$ (replace $\ell-1$ by $2 \ell-1$ and by $2 \ell-2$ in the case type B and D, respectively) such that $\theta_{j}^{\prime} \notin D^{(2)}(S)^{W}$.

Suppose that $\theta_{j}^{\prime} \in D^{(2)}(S)^{W}$ for any $j$. Since $C_{\mathcal{A}}$ is a basis for $D^{(2)}(\mathscr{A})$ by Theorem 4.4, we may write

$$
\theta_{j}^{\prime}=\sum_{\lambda} f_{\lambda} \theta_{\lambda}^{\mathcal{A}}+\sum_{k=1}^{\ell} a_{k} \eta_{k}^{\mathcal{A}}
$$


for some $f_{\lambda} \in S$ and $a_{k} \in \mathbb{R}$. For any $w \in W$, we have

$$
w \cdot \theta_{j}^{\prime}=\sum_{\lambda}\left(w \cdot f_{\lambda}\right) \theta_{\lambda}^{\mathcal{A}}+w \cdot \sum_{k=1}^{\ell} a_{k} \eta_{k}^{\mathcal{A}}
$$

by Lemma 6.2. Since $w \cdot \theta_{j}^{\prime}=\theta_{j}^{\prime}$ and $C_{\mathcal{A}}$ is linearly independent over $S$, we have that $f_{\lambda}$ is $W$-invariant for $\lambda \in \Lambda$. Then

$$
\sum_{k=1}^{\ell} a_{k} \eta_{k}^{\mathcal{A}} \in D^{(2)}(S)^{W}
$$

By Lemma 6.3, the vector space $\left\langle\eta_{1}^{\mathcal{A}}, \ldots, \eta_{\ell}^{\mathcal{A}}\right\rangle_{\mathbb{R}} / \mathbb{R}\left(\eta_{1}^{\mathcal{A}}+\cdots+\eta_{\ell}^{\mathcal{A}}\right)$ is a nontrivial irreducible representation. Thus we have $a_{1}=\cdots=a_{\ell}$ (replace it by $a_{1}=0, \ldots, a_{\ell}=0$ in the case of type $\mathrm{B}$ and $\left.\mathrm{D}\right)$. This leads that $\theta_{1}^{\prime}, \ldots, \theta_{s_{2}}^{\prime}$ is linearly dependent over $S$. This is a contradiction.

\section{Acknowledgements}

The author thanks Professor Soichi Okada for his helpful comments.

\section{References}

[1] N. Bourbaki, Groupes et Algèbres de Lie. Chapitres 4,5 et 6, Hermann, Paris, (1968).

[2] P. Holm, Differential Operators on Arrangements of Hyperplanes. PhD. Thesis, Stockholm University, (2002).

[3] P. Holm, Differential Operators on Hyperplane Arrangements. Comm. Algebra 32 (2004), no.6, 2177-2201.

[4] J. E. Humphreys, Reflection Groups and Coxeter Groups. Cambridge Univ. Press, Cambridge, New York (1990).

[5] M. Ito and S. Okada, An Application of Cauchy-Sylvester's Theorem on Compound Determinants to a $B C_{n}$-Type Jackson Integral. Partitions, Q-Series, and Modular Forms (2012), Volume 23, 145-157. 
[6] T. Józefiak and B. E. Sagan, Basic derivations for subarrangements of Coxeter arrangements. J. Algebraic Combin. 2 (1993), no.3, 291-320.

[7] J. C. McConnell and J. C. Robson, Noncommutative Noetherian Rings. Pure and Applied Mathematics, John Wiley \& Sons, Chichester, 1987.

[8] N. Nakashima, The Neotherian properties of the rings of differential operators on central 2-arrangements. Communications in algebra, to appear.

[9] N. Nakashima, Bases for modules of differential operators of order 2 on the classical Coxeter arrangements. Proceeding of 24th International Conference on Formal Power Series and Algebraic Combinatorics (FPSAC), Nagoya (2012), submitted.

[10] P. Orlik and H. Terao, Arrangements of Hyperplanes. Grundlehren dermatematischen Wissenschaften 300, Springer-Verlag, 1992.

[11] K. Saito, Theory of logarithmic differential forms and logarithmic vector fields. J. Fac. Sci. Univ. Tokyo Sect. IA Math. 27 (1980), 265-291. 